\title{
Publisher's Note: Stability of an emittance-dominated sheet-electron beam in planar wiggler and periodic permanent magnet structures with natural focusing [Phys. Rev. ST Accel. Beams 8, 062001 (2005)]
}

B. E. Carlsten, L. M. Earley, F. L. Krawczyk, S. J. Russell, J. M. Potter, P. Ferguson, and S. Humphries, Jr. (Received 9 November 2005; published 17 November 2005)

DOI: 10.1103/PhysRevSTAB.8.119902

PACS numbers: $41.85 .-\mathrm{p}, 99.10 . \mathrm{Fg}$

This paper was published online on 29 June 2005 with formatting errors in Ref. [16]. Reference [16] should read as "B. E. Carlsten, L. M. Earley, F. L. Krawczyk, S. J. Russell, J. M. Potter, P. Ferguson, and S. Humphries, Jr., this issue, Phys. Rev. ST Accel. Beams 8, 062002 (2005)." The reference has been corrected as of 10 November 2005. 